> La découverte de petites protéines globulaires, capables de se lier à des molécules odorantes, dans la lymphe sensillaire des antennes des insectes et dans le mucus nasal des mammifères, a conduit au concept selon lequel les «protéines liant les odeurs » (odorant binding proteins, OBP) et les protéines chimiosensorielles (chemosensory proteins, (SP) pourraient optimiser la réception des stimulus - molécules hydrophobes - en facilitant leur solubilisation dans l'environnement aqueux qui entoure les neurones sensoriels. L'acquisition d'OBP représenterait une des adaptations moléculaires à la vie terrestre, un phénomène qui, au vu de la divergence des $O B P$, aurait évolué indépendamment chez les insectes et chez les mammifères. L'étude des propriétés fonctionnelles, des variations individuelles et intra-spécifiques, et des mécanismes réglant l'expression de ces protéines constitue une voie possible pour aider non seulement au développement de nouvelles méthodes pour le contrôle des insectes nuisibles, mais également à la compréhension des bases moléculaires de l'olfaction et de certaines anosmies. <

\section{Les péri-récepteurs chimiosensoriels des insectes}

Jean-François Picimbon

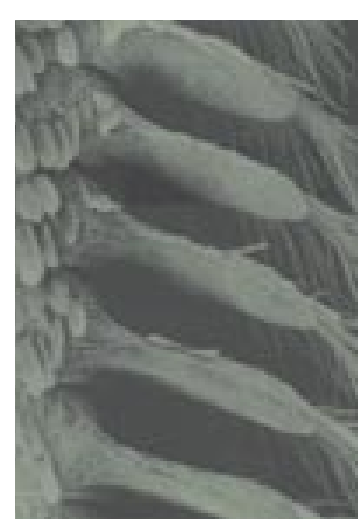

Département d'Écologie,

Université de Lund, sölvegatan 37, SE-223 62 Lund, Suède.

jean-francois.picimbon@ekol.lu.se

libère une phéromone sexuelle qui attire à distance uniquement les mâles conspécifiques. La sensibilité et la sélectivité du système biologique sont remarquables: une seule molécule de phéromone suffit à déclencher une réponse physiologique et comportementale. L'exploration du système sensoriel olfactif des insectes est donc un modèle expérimental particulièrement approprié pour étudier les principes fondamentaux de la chimiosensibilité et mieux comprendre des systèmes olfactifs plus complexes comme ceux des vertébrés les plus évolués.

Tous les organismes vivants se doivent de connaître le monde environnant afin d'y évoluer et de s'y reproduire, ou tout simplement d'y survivre. Pour analyser le monde qui les entoure, tous les animaux possèdent des facultés sensorielles qui leur permettent de communiquer avec lui. L'olfaction et le goût jouent un rôle vital chez les insectes; ils recourent à des signaux chimiques dans la détection de nourriture, de nid ou de site d'oviposition*, dans l'établissement de relations inter-individuelles, sociales et sexuelles, et dans l'appréhension du danger (reconnaissance d'un prédateur, d'une substance toxique). Par exemple, les papillons de nuit ont développé une communication chimique performante qui leur permet d'identifier si un individu appartient à la même espèce ou non: la femelle

* Dépôt des œufs par l'insecte femelle.

\section{Les sensilles: les microcapteurs chimiosensoriels}

La détection des molécules odorantes chez les insectes met en jeu des structures particulières, les antennes et les sensilles, qui représentent des micro-organes (ou organules) sensoriels véritablement programmés pour l'olfaction. Ils recouvrent les branches antennaires par milliers et fonctionnent comme des microcapteurs périphériques des molécules odorantes de l'air environnant. II existe différents types sensillaires mais tous ont une architecture commune, un système de pores tubulaires qui connecte le milieu extérieur à la lumière sensillaire renfermant les dendrites des neurones sensoriels (deux à trois cellules sensorielles par sensille). En coupe transversale, les pores cuti- 
culaires et l'entrée des molécules odorantes sont séparés des cellules nerveuses cibles par un fluide aqueux protecteur, la «lymphe sensillaire ». Cette lymphe, équivalent chez les insectes du mucus nasal des mammifères, constitue une véritable barrière pour les molécules odorantes très hydrophobes.

Chaque sensille répond de façon spécifique à une molécule chimique ou à une famille de molécules chimiques, ce qui explique la grande diversité morphologique. Sur la base de leur spécificité chimiosensorielle, on reconnaît au moins cinq types de sensilles olfactifs: (1) les sensilles trichodéiques, larges, épais et allongés (25-35 mm), impliqués dans la réception des molécules de phéromone sexuelle et largement répandus sur l'antenne des mâles chez les papillons de nuit (Figures 1 et 2); (2) les sensilles basiconiques, plus petits $(15-25 \mathrm{~mm})$, présents dans les deux sexes chez une grande variété d'insectes, impliqués dans la reconnaissance de molécules odorantes généralistes (les molécules volatiles des plantes, des œufs ou des larves); (3) les sensilles placodéiques ou plaques olfactives des antennes d'abeilles et de scarabées; (4) les sensilles cœloconiques en « pince à linge »; et (5) les sensilles chaétiques; ces deux derniers types, répartis dans différentes parties du corps de l'insecte, sont rencontrés chez les papillons de nuits, chez les abeilles et dans d'autres familles d'insectes, et sensibles aux molécules odorantes ou gustatives, au gaz carbonique, à la température, à l'humidité ou à une combinaison de ces différentes modalités chimiques. Le répertoire sensillaire des insectes est donc très varié, répondant parfaitement à la diversité des stimulus chimiques et au nombre infini de molécules odorantes et gustatives.

\section{Les protéines se liant aux odeurs: odorant-binding proteins}

Pour traverser la lymphe et atteindre les neurones sensoriels, des mécanismes périphériques de solubilisation des molécules odorantes sont nécessaires. De petites protéines acides, très concentrées dans la lymphe, permettraient la solubilisation des molécules odorantes. Ces protéines qui se lient aux molécules odorantes à la périphérie des neurones olfactifs sont appelées odorant-binding proteins (OBP) [1]. Les OBP sont des chaînes polypeptidiques simples (environ 150 acides aminés) caractérisées par six cystéines reliées par trois ponts disulfures, donnant aux OBP une structure spécifique [2, 3]. Les OBP auraient de multiples fonctions (Figure 2C). Lorsque les tubules cuticulaires sont au contact direct des neurones sensoriels à l'intérieur du sensille, les molécules odorantes débouchant du tubule activeraient les récepteurs moléculaires olfactifs et les OBP agiraient en inhibiteurs précoces, libérant le récep-
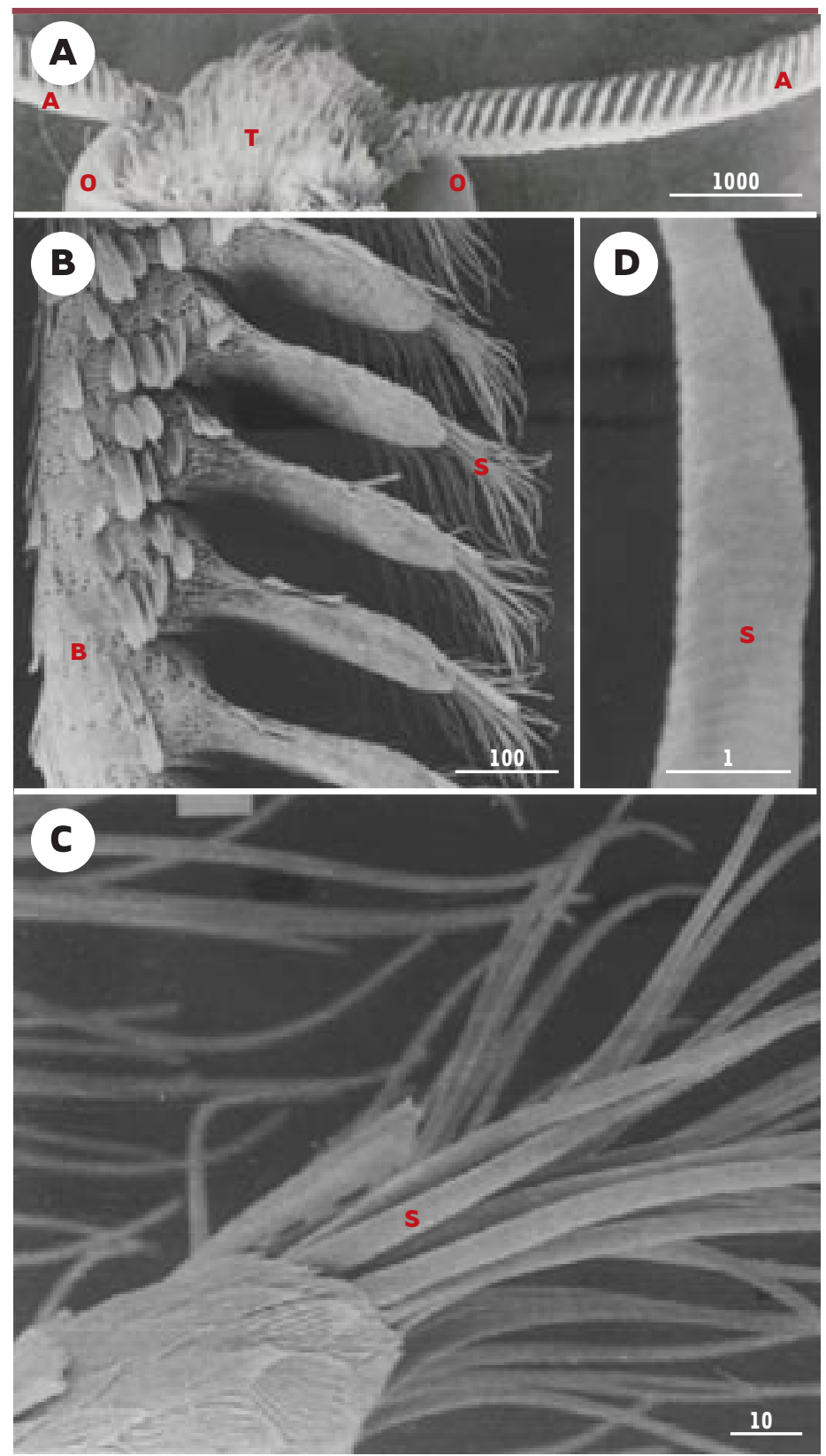

Figure 1. Antenne du papillon de nuit mâle observée en microscopie électronique en balayage. A. Aspect général des antennes (agrandissement: x 50, échelle: $1000 \mu \mathrm{m}$ ); A: antenne, 0: œil, T: tête. B. Organisation de l'antenne (agrandissement: $x 360$, échelle: $100 \mu \mathrm{m})$; B : tronc antennaire; S: sensilles olfactifs. C. Vue rapprochée des sensilles olfactifs à l'extrémité d'une branche antennaire (agrandissement: $x 50$, échelle : $10 \mu \mathrm{m}$ ). $D$. très fort grossissement d'un sensille olfactif, siège des dendrites des neurones sensoriels (agrandissement: $x 50000$, échelle: $1 \mu \mathrm{m}$ ). Les antennes des mâles de papillon de nuit apparaissent comme des structures très ramifiées, parcourues par le nerf antennaire et dont les branches portent des dizaines de milliers de cils chimiosensoriels (soies ou sensilles). Les sensilles olfactifs apparaissent comme des extensions cuticulaires allongées dont la surface, poreuse, permet l'entrée des molécules odorantes volatiles. 
teur des molécules odorantes et présentant ces molécules aux enzymes de dégradation (modèle de contact) [4]. Lorsque les canaux tubulaires s'étendent à l'intérieur du sensille à distance des neurones et délivrent les molécules odorantes directement dans la lymphe, les OBP pourraient prendre en charge le transport des molécules odorantes jusqu'aux récepteurs olfactifs. Une fois que les molécules odorantes ont pénétré à l'intérieur du sensille, plusieurs interactions sont alors possibles, conduisant soit à l'activa-

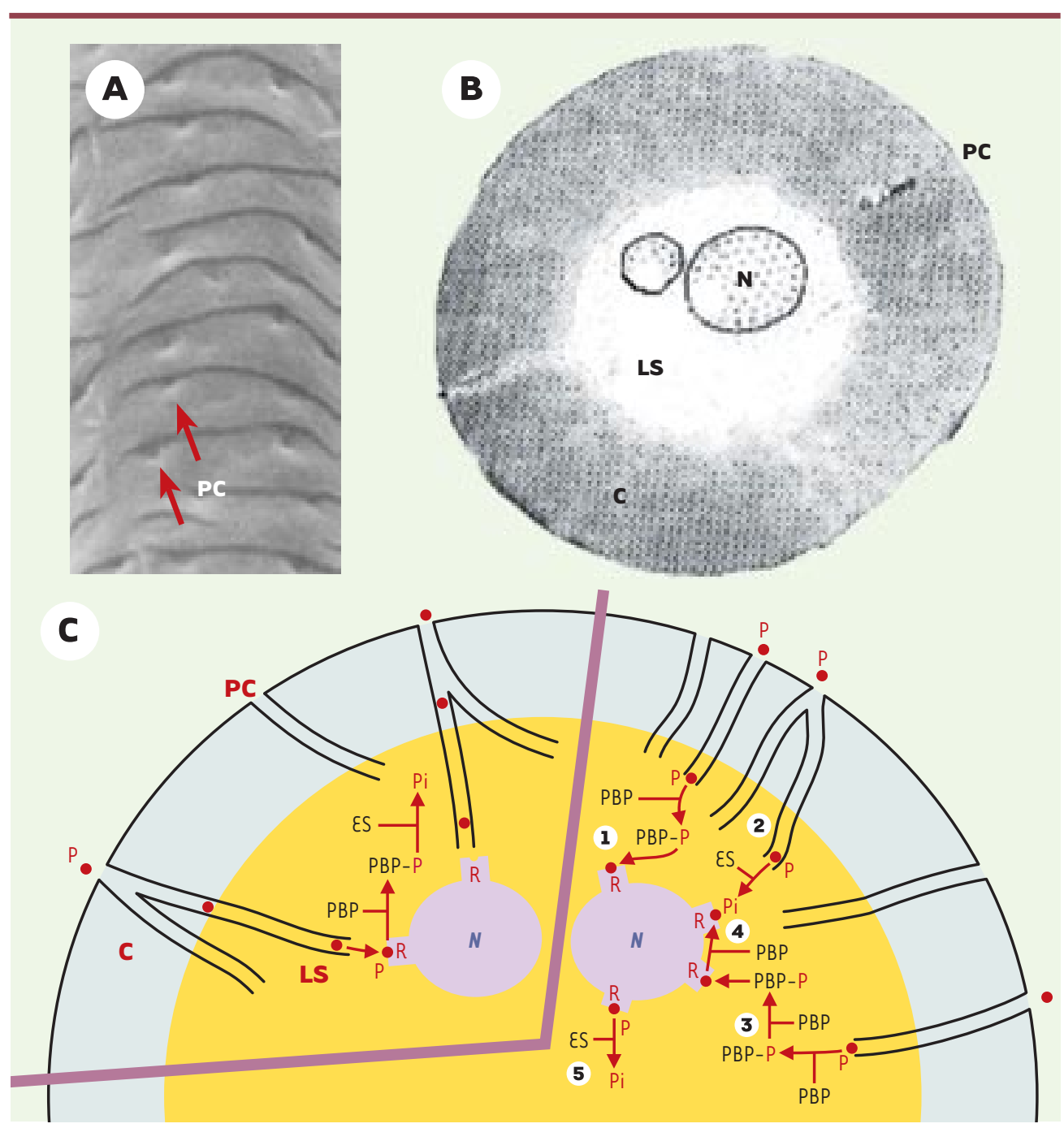

Figure 2. Organisation structurale et moléculaire des sensilles olfactifs. A. Vue rapprochée des pores cuticulaires (PC) d'un sensille olfactif (agrandissement: x 40000). B. sensille olfactif en coupe transversale (agrandissement: $x 25000) \star$. C. Modèles biochimiques de la réception de phéromone à la phériphérie des neurones sensoriels d'un sensille olfactif (représenté schématiquement en coupe transversale). Chaque sensille renferme en général deux neurones olfactifs spécialisés $(\mathrm{N})$ dont les dendrites s'étendent dans les prolongements cuticulaires et portent les structures perceptives protégées par un fluide aqueux, la lymphe sensillaire (LS). Au contact de l'antenne, les molécules odorantes (phéromone, $P$ ) sont absorbées à la surface cireuse de ces sensilles et diffusent à travers les pores de la paroi cuticulaire $(C)$ pour atteindre la lumière sensillaire. Dans le modèle de contact, la phéromone atteint le récepteur membranaire dendritique $(R)$ par diffusion à travers les pores cuticulaires $(P C)$. La désactivation du récepteur est réalisée par fixation de la molécule de phéromone à la pheromone binding protein (PBP) qui la présente à l'enzyme de dégradation de la phéromone, l'estérase sensillaire (ES). Dans le modèle d'équilibre cinétique, la molécule de phéromone peut se lier soit à une PBP qui la véhicule jusqu'au site récepteur (1), soit à une enzyme de dégradation qui l'inactive (Pi pour phéromone inactive) (2), soit interagir avec de multiples PBP avant d'atteindre le récepteur membranaire (3). Après activation du récepteur, la phéromone est déplacée par une PBP et peut aller activer un autre récepteur (4) ou est dégradée par l'estérase sensillaire (5). *Adapté de [27]. tion du récepteur, soit à I'inactivation et à l'élimination de la molécule odorante par une enzyme de dégradation, l'estérase sensillaire (modèle de l'équilibre cinétique). Dans ce modèle, I'OBP pourrait se lier plusieurs fois à une molécule odorante avant ou après l'interaction de celle-ci avec le récepteur et une molécule odorante pourrait interagir avec plusieurs récepteurs avant d'être inactivée. Ce schéma assigne donc aux OBP des propriétés multifonctionnelles: solubilisation, transport et protection vis-à-vis des enzymes de dégradation [5]. Chez les insectes, les OBP seraient plus que de simples transporteurs ( $\mathrm{Fi}$ gure 3). Elles participeraient activement au filtrage et à la reconnaissance des signaux olfactifs à la périphérie des neurones sensoriels. Chaque OBP aurait un répertoire de ligand bien établi avec une affinité déterminée pour une molécule odorante ou une série chimique spécifique $[6,7]$.

Différents groupes d'OBP ont été identifiés en fonction des molécules odorantes auxquelles ils se lient (Tableau I) [8-15]. Des protéines appartenant à la famille des OBP sont également présentes dans les sensilles gustatifs. Chez la mouche Phormia regina, l'application d'anticorps dirigés contre ces pro- 
téines entraîne une diminution de la réponse électrophysiologique des neurones gustatifs à certains stimulus chimiomosensoriels. Cette expérience démontre le rôle important des molécules de type OBP dans le transport non seulement des molécules odorantes mais aussi des molécules gustatives [16].

\section{Les protéines chimiosensorielles: chemosensory proteins}

Récemment, Picimbon et al. [17, 18] ont découvert que les sensations chimiques de contact impliqueraient une autre classe de protéines: les protéines chimiosensorielles (chemosensory protein, CSP). Ces protéines ont été identifiées chez la plupart des insectes, des papillons de nuits aux phasmes [19-21]. Dans chaque espèce, l'existence de plusieurs types de CSP au sein d'un même individu a été démontrée, ce qui suggère que les CSP pourraient avoir de multiples fonctions. Le rôle des CSP dans la sensation chimique générale de contact est fortement suggéré par leur expression sur tout le corps de l'insecte mais en particulier sur les pattes. L'ontogénie de ces protéines est décalée par rapport au développement du système olfactif et à la synthèse d'OBP. Elle est plutôt en phase avec le développement de la cuticule [22]. La structure des CSP est également très différente de celle des OBP: ce sont des chaînes polypeptidiques d'environ 110 acides aminés dont quatre cystéines reliées par deux ponts disulfures (Cys 29-38, Cys 55-59). Au contraire des OBP, la structure primaire des CSP est très conservée chez les différentes espèces d'insectes et leur structure secondaire forme des structures en boucles semblables à celles des thiorédoxines. Les CSP pourraient ainsi se lier à de multiples ligands, le $\mathrm{CO}_{2}$ et des molécules gustatives comme divers sucres et sels.

\section{Chez les mammifères}

L'olfaction est un don inné qui repose sur des bases moléculaires conservées de l'insecte à l'homme. Les OBP/CSP ne seraient pas spécifiques des insectes; Pelosi et al. ont découvert, dans le mucus olfactif de la vache, une protéine capable de se lier à une molécule odorante du poivre, le 2isobutyl-3-méthoxypyrazine [23]. La structure de la protéine se liant à la pyrazine diffère fortement de celle des protéines se liant aux molécules odorantes des insectes mais évoque celle des lipocalines, une famille de protéines transporteurs de molécules hydrophobes en milieu aqueux. II semble donc que certaines protéines du mucus olfactif pourraient avoir une fonction semblable aux OBP des insectes, à savoir faciliter la solubilisation et le transport des molécules odorantes. Cependant, chez les mammifères, le rôle des $O B P$ ne semble pas crucial; les résultats montrent qu'après délétion du gène codant pour l'OBP, les animaux ne sont pas anosmiques et que l'on peut observer des réponses électriques induites par des molécules odorantes dans l'épithélium olfactif des animaux transgéniques. De plus, toutes les expériences réalisées in vitro montrent que les $\mathrm{OBP}$ ne sont pas indispensables pour induire une réponse des neurones sensoriels. L'étude électrophysiologique des neurones olfactifs isolés (patch-clamp, configuration cellule entière) a permis d'enregistrer des courants ioniques qui se développent en réponse à l'application de stimulus odorants [24]. Par ailleurs, des récepteurs olfactifs « chimères » exprimés dans des cellules HEK 293 (human embryonic kidney) répondent sélectivement à des molécules odorantes différentes [25]. L'ensemble de ces expériences suggère fortement que la reconnaissance des molécules odorantes par les récepteurs olfactifs représenterait le premier niveau de discrimination de l'odeur et que les OBP

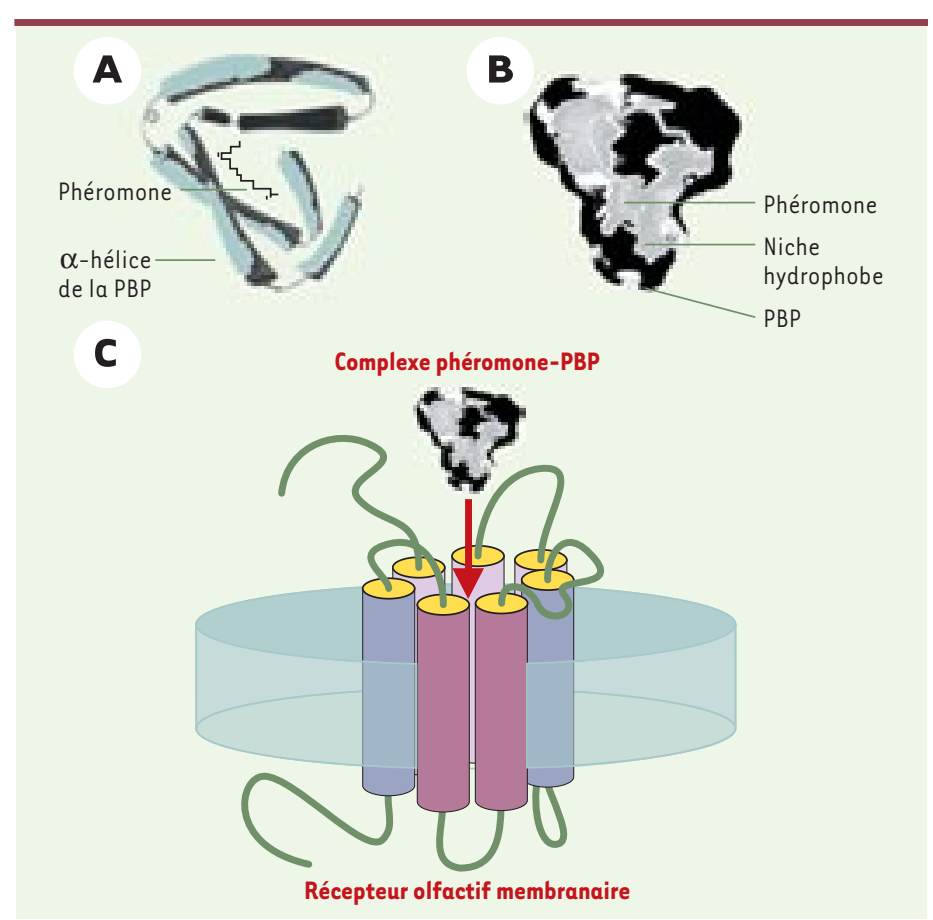

Figure 3. Structure moléculaire fonctionnelle de la protéine se liant à la phéromone. A. Modèle de la structure moléculaire de la PBP prédit par Breer et al. [2]. B. Cristal du complexe PBP-phéromone du bombyx du mûrier. D’après [3]. C. Possible mécanisme d'activation du récepteur sensoriel: le complexe PBPphéromone niché dans le récepteur olfactif à sept domaines transmembranaires. La résolution de la structure tertiaire de la PBP du bombyx du mûrier confirme les prédictions de Breer et al., une niche hydrophobe constituée par sept hélices antiparallèles de type $\alpha$ qui englobe la molécule de phéromone. Cette « encapsulation » de la molécule de phéromone permettrait à la molécule d'être transportée jusqu'aux récepteurs sensoriels, d'être protégée des enzymes de dégradation et même de réaliser un complexe activateur des récepteurs olfactifs. Les CSP, dont la taille et les propriétés physiques (acidité, nombre d'hélices $\alpha$ ) sont proches de celles des OBP, pourraient encapsuler semblablement les molécules gustatives. 
ne joueraient aucun rôle dans la discrimination olfactive. Malgré la découverte de gènes codant pour les OBP chez l'homme, le rôle des $\mathrm{OBP}$ chez les mammifères reste très hypothétique et n'est probablement pas lié à la reconnaissance spécifique de molécules odorantes. De plus, si la capacité de reconnaître une odeur est fondamentale pour la survie de l'insecte, pour la détection du danger et de la nourriture, il en est tout autrement pour l'homme qui évolue dans un environnement socio-culturel qui le protège. Néanmoins, n'oublions jamais que la perte de l'odorat peut avoir de profondes conséquences psychologiques et somatiques. Une odeur stimulera ou améliorera la vie d'un individu aussi longtemps qu'elle évoquera un parfum, un événement, une émotion ou une personne.

Au total, les organes sensoriels olfactifs des insectes représentent sans aucun doute un des meilleurs systèmes quant au rapport signal/bruit (c'est-à-dire l'extraction d'un signal spécifique de l'environnement extérieur). Dans le cadre de la communication phéromonale et de la rencontre des partenaires sexuels chez les insectes, la femelle émet des quantités infimes d'un mélange spécifique de molécules informatives; la réponse du mâle repose sur la reconnaissance sélective de ce signal qui lui parvient dans un spectre complexe de molécules odorantes. Des filtres périphériques, œuvrant comme de véritables microcapteurs chimiosensoriels, permettent d'extraire le signal et d'éliminer les interférences que représentent les odeurs non spécifiques produites par des individus d'une autre espèce ou d'autres odeurs de l'environnement. Le fonctionnement des microcapteurs olfactifs reposerait non seulement sur les récepteurs moléculaires des neurones primaires sensillaires répondant seulement à quelques composés même à faible concentration, mais aussi sur les pheromone binding pro- teins (PBP), qui sont des OBP impliquées dans la capture des molécules de phéromone sexuelle. La compréhension de la reconnaissance par la PBP sensillaire des molécules de phéromone pourrait offrir de nouvelles potentialités économiques et industrielles dans le cadre du contrôle des insectes nuisibles et de la mise au point de «nez artificiels » ainsi que de nouvelles voies de traitements cliniques des troubles de l'olfaction et du goût. Ces maladies sont le plus souvent associées à un état dépressif et à l'allergie. L'utilisation de protéines de type lipocaline, OBP ou CSP, pourrait permettre de modifier l'olfaction ou le goût et de corriger non seulement des désordres sensoriels divers (hyposmie, parosmie, agueusie, dysgueusie...) mais aussi des problèmes physiologiques touchant l'apport de substances énergétiques ou de signaux essentiels pour les cellules de l'organisme. Ces protéines pourraient être utilisées pour délivrer des agents antagonistes des récepteurs du goût et de l'olfaction ou pour séquestrer des molécules odorantes et gustatives, ce qui induirait une diminution du fonctionnement des effecteurs sensoriels. Cette voie reste à explorer. Par ailleurs, d'un point de vue agronomique, bloquer spécifiquement les OBP et les CSP serait un moyen efficace de bloquer le système olfactif et chimiosensoriel des insectes ravageant les récoltes. Des études comparatives analysant les relations structure/activité des ligands olfactifs pourraient conduire à des molécules inhibant spécifiquement l'olfaction de l'insecte, captées par les PBP et interagissant avec les récepteurs. Ces molécules inhibitrices pourraient aussi hyperactiver les enzymes de dégradation ou les inhiber, aboutissant dans les deux cas à un dérèglement du système de sensibilisation-désensibilisation du récepteur. Les PBP constitueraient donc des cibles moléculaires potentielles pour la lutte intégrée contre les insectes nuisibles [26]. $\diamond$

\begin{tabular}{|c|c|c|c|c|c|c|c|}
\hline & PBP & GOBP & ABP & PBP-RP & LUSH & ASP & CSP \\
\hline Insecte & Papillon de nuit & Papillon de nuit & Papillon de nuit & Mouche & Mouche & $\begin{array}{l}\text { Abeille } \\
\text { sauterelle, } \\
\text { mouche, blattes }\end{array}$ & Papillon de nuit \\
\hline $\begin{array}{l}\text { Poids moléculaire } \\
(k D a)\end{array}$ & $15,7-16,5$ & 17,0 & 14,5 & $14,1-14,4$ & 14,0 & 13,1 & $12-13$ \\
\hline Sensille & $\begin{array}{l}\text { Olfactif } \\
\text { basiconica }\end{array}$ & Olfactif & $\begin{array}{l}? \\
\text { trichodea }\end{array}$ & $\begin{array}{l}\text { Olfactif } \\
\text { trichodea }\end{array}$ & $\begin{array}{l}\text { Chimiosensible } \\
\text { placodea }\end{array}$ & $\begin{array}{l}\text { Olfactif } \\
\text { cheticum }\end{array}$ & $\begin{array}{l}\text { Chimiosensible } \\
\text { de contact }\end{array}$ \\
\hline Ligand & $\begin{array}{l}\text { Phéromone } \\
\text { sexuelle }\end{array}$ & $\begin{array}{l}\text { Molécules } \\
\text { odorantes } \\
\text { des plantes }\end{array}$ & $\begin{array}{l}? \\
?\end{array}$ & $\begin{array}{l}? \\
?\end{array}$ & Éthanol & $\begin{array}{l}\text { Phéromone } \\
\text { de Reine }\end{array}$ & $\begin{array}{l}\text { Molécules chimiques } \\
\text { hydrophobes } \\
\text { de contact, sucre, } \\
\mathrm{CO}_{2}, \ldots\end{array}$ \\
\hline Références & [1-8] & {$[9,10]$} & {$[11,12]$} & [13] & [14] & [15] & [17-22] \\
\hline
\end{tabular}

Tableau I. Groupes de protéines se liant aux molécules odorantes (OBP) et aux protéines chimiosensorielles (CSP) chez les insectes. PBP: pheromone binding protein ; GOBP: general odorant binding protein; ABP: antennal binding protein ; PBP-RP: pheromone binding protein-related protein ; ASP: antennal-specific proteins; LUSH: produit du gène mutant lush; CSP: chemosensory protein. 


\section{SUMMARY}

\section{Chemosensory perireceptors of insects}

Small globular proteins, highly abundant in the antennal sensillar lymph and in the mammalian nasal mucus, and capable of binding odorant molecules have been discovered. They have led to the concept that odorant-binding proteins (OBPs) and chemosensory proteins (CSPs) may optimize the reception of hydrophobic stimuli molecules by enhancing their solubilization in the aqueous environment that bath the sensory neurons. The acquisition of OBP may represent one of the molecular adaptations to terrestrial life, a phenomenon that may have occured independently in insects and mammals with respect to the great dissimilarity observed in their OBPs. Studying OBP with regard to the functional properties, the individual and interspecific variations and the regulatory mechanisms of synthesis offer new potentials, not only in the development of novel methods to control insect pests but also to the understanding of the molecular basis of olfaction and specific anosmia. $\diamond$

\section{RÉFÉRENCES}

1. Pelosi P. Odorant-binding proteins. Crit Rev Biochem Mol Biol 1994; 29: 199-228.

2. Breer H, Boekhoff I, Krieger J, et al. Molecular mechanisms of olfactory signal transduction. In: Corey DP, Roper SD, eds. Sensory transduction. New York: Rockefeller University Press, 1992: 94-108.

3. Sandler BH, Nikonova L, Leal WS, et al. Sexual attraction in the silkworm moth: structure of the pheromonebinding-protein-bombykol complex. Chem Biol 2000; 7 . 143-51.

4. Vogt RG, Riddiford LM. Pheromone binding and inactivation by moth antennae. Nature 1981; 293: 161-3.

5. Vogt RG, Riddiford LM. Pheromone reception: a kinetic equilibrium. In: Payre $T L$, Birch MC, Kennedy (E), eds. Seminar on mechanisms in perception and orientation to insect olfactory signals. Oxford: Clarendon Press, 1986: 201-8.

6. Du G, Ng CS, Prestwich GD. Odorant binding by a pheromone binding protein-
11. Vogt RG, Callahan FE, Rogers $M E$, et al. Odorant binding proteins diversity and distribution among the insect orders, as indicated by LAP, an OBP-related protein of the true bug Lygus lineolaris (Hemiptera, Heteroptera). Chem Senses 1999; 24: 481-95.

12. Krieger J, von NickischRoseneck EV, Mameli M. et al. Binding proteins from the antennae of Bombyx mori. Insect Biochem Mol Biol 1996; 26: 297-307.

13. Danty $\varepsilon$, Briand L, MichardVanhee $C$, et al. Cloning and expression of a queen pheromone binding protein in the honeybee: an olfactoryspecific, developmentally regulated protein. J Neurosci 1999; 1: 7468-75.

14. Kim MS, Repp A, Smith DP. LUSH odorant-binding protein mediates chemosensory responses to alcohols in Drosophila melanogaster. Genetics 1998; 150: 711-21.

15. McKenna MP, Hekmat-Scafe DS, Gaines $P$, et al. Putative Drosophila pheromonebinding-proteins expressed in a subregion of the olfactory system. J Biol Chem 1994; 269: 16340-7.

16. Ozaki M, Morizaki K, Idei W, et al. A putative lipophilic stimulant carrier protein commonly found in the taste and olfactory systems $A$ unique member of the pheromone binding protein superfamily. Eur / Biochem 1995; 230: 298-308.

17. Picimbon JF, Leal WS. Olfactory soluble proteins of cockroaches. Insect Biochem Mol Biol 1999; 29: 973-8.

18. Picimbon JF, Dietrich K, Breer $\mathrm{H}$, et al. Chemosensory proteins of Locusta migratoria (Orthoptera: Acrididae). Insect Biochem Mol Biol 2000; 30: 233-41.

19. Angeli S, Ceron F, Scaloni A, et al. Purification, structural characterization, cloning and immunocytochemical localization of chemoreception proteins from Schistocerca gregaria. Eur J Biochem 1999; 262: 745-54.
20. Picimbon JF, Dietrich K, Angeli $S$, et al. Purification and molecular cloning of chemosensory proteins from Bombyx mori. Arch Insect Biochem Physiol 2000; 44 : 120-9.

21. Marchese S, Angeli S, Andolfo A, et al. Soluble proteins from chemosensory organs of Eurycantha calcarata (insects, Phasmatodea). Insect Biochem Mol Biol 2000; 30: 1091-8.

22. Picimbon JF, Dietrich K, Krieger J, et al. Identity and expression pattern of chemosensory proteins in Heliothis virescens (Lepidoptera, Noctuidae). Insect Biochem Mol Biol 2001; 29: 973-8.

23. Pelosi P, Baldaccini NE, Pisanelli AM. Identification of a specific olfactory receptor for 2-isobutyl-3methoxypyrazine. Biochem J 1982; 201: 245-8.

24. Kurahashi T, Menini A. Mechanism of odorant adaptation in the olfactory receptor cell. Nature 1997; 385: 725-9.

25. Krautwurst D, Yau KW, Reed RR. Identification of ligands for olfactory receptors by functional expression of a receptor library. Cell 1998; 95: 917-26.

26. Picimbon JF. Protéines liant les odeurs (OBP) et protéines chimiosensorielles (CSP): cibles moléculaires de la lutte intégrée. In: RegnaultRoger C, Philogène B, Vincent $C$, eds. Biopesticides d'origine végétale. Paris: Lavoisier Tec et Doc, 2002 : 265-83.

27. Steinbrecht RA. Olfactory receptors. In: Eguchi $\varepsilon$, Tominaga y, eds. Atlas of arthropod sensory receptors, dynamic morphology in relation to function. Tokyo, Japon : Springer-Verlag, 1999 : 155-76.

\section{TIRÉS À PART} and Bombyx. Cell Tissue Res 1995; 282: 203-17. 